\title{
Preparation of polylactide-co-glycolide nanoparticles incorporating celecoxib and their antitumor activity against brain tumor cells
}

This article was published in the following Dove Press journal:

International Journal of Nanomedicine

27 October 20II

Number of times this article has been viewed

\author{
Tae-Ho Kim ${ }^{*}$ \\ Young-II Jeong ${ }^{2 *}$ \\ Shu-Guang Jin ${ }^{2}$ \\ Jian $\mathrm{Pei}^{2}$ \\ Tae-Young Jung' \\ Kyung-Sub Moon' \\ In-Young Kim' \\ Sam-Suk Kang' \\ Shin Jung ${ }^{1,2}$ \\ 'Department of Neurosurgery, \\ ${ }^{2}$ Brain Tumor Research Laboratory, \\ Chonnam National University \\ Research Institute of Medical Science, \\ Chonnam National University \\ Hwasun Hospital and Medical School, \\ Gwangju, Republic of Korea \\ *These authors contributed equally to \\ this work.
}

Correspondence: Shin Jung

Department of Neurosurgery, Chonnam National University Hwasun Hospital, I 60 llsim-ri, Hwasun-eup, Hwasun-gun, Jeollanam-do 519-809, Republic of Korea $\mathrm{Tel}+82613797666$

Fax +82 6I 3797673

Email sjung@chonnam.ac.kr
Background: Celecoxib, a cyclo-oxygenase (COX)-2 inhibitor, has been reported to mediate growth inhibitory effects and to induce apoptosis in various cancer cell lines. In this study, we examined the potential effects of celecoxib on glioma cell proliferation, migration, and inhibition of COX-2 expression in vitro.

Methods: Celecoxib was incorporated into poly DL-lactide-co-glycolide (PLGA) nanoparticles for antitumor drug delivery.

Results: PLGA nanoparticles incorporating celecoxib had spherical shapes and their particle sizes were in the range of 50-200 $\mathrm{nm}$. Drug-loading efficiency was not significantly changed according to the solvent used, except for acetone. Celecoxib was released from the PLGA nanoparticles for more than 2 days, and the higher the drug content, the longer the duration of drug release. PLGA nanoparticles incorporating celecoxib showed cytotoxicity against U87MG tumor cells similar to that of celecoxib administered alone. Furthermore, celecoxib did not affect the degree of migration of U87MG cells. PLGA nanoparticles incorporating celecoxib showed dose-dependent cytotoxicity similar to that of celecoxib alone in C6 rat glioma cells. Western blot assay of the $\mathrm{C} 6$ cells showed that neither celecoxib alone nor PLGA nanoparticles incorporating celecoxib affected COX-2 expression.

Conclusion: PLGA nanoparticles incorporating celecoxib had antitumor activity similar to that of celecoxib alone, even though these particles did not affect the degree of migration or COX-2 expression in the tumor cells.

Keywords: celecoxib, cyclo-oxygenase-2, PLGA nanoparticles, glioma, antitumor activity

\section{Introduction}

Cyclo-oxygenase (COX)-2 is the key enzyme in arachidonic acid metabolism resulting in prostaglandin production, and is induced by several factors, such as growth factors, cytokines, and tumor promoters. It is thus an inducible enzyme responsible for prostaglandin production at sites of inflammation and is involved in cell proliferation. ${ }^{1-5}$ In particular, COX-2 expression and prostaglandin production are associated with tumorigenesis and tumor progression. Expression of COX-2 has been reported to be associated with the complex changes observed in a variety of diseases of the brain. Following trauma, an increase in prostaglandin levels can lead to vascular damage. In glioblastoma cells, inhibitors of eicosanoid biosynthesis suppress proliferation and promote astrocytic differentiation. ${ }^{6} \mathrm{COX}-2$ expression has also been detected in colorectal, gastric, esophageal, and lung carcinomas, as well as in brain tumors. ${ }^{7-13}$ COX-2 has been reported to be important in the relationship between increased prostaglandin synthesis and the development of glioma and its progression. ${ }^{14,15}$ 
Celecoxib, a selective COX-2 inhibitor, has been reported to mediate growth inhibitory effects and to induce apoptosis in various cancer cell lines. ${ }^{10-13}$ Selective COX-2 inhibitors, such as celecoxib, have attracted interest as being safe and effective anticancer agents. The aim of this study was to encapsulate celecoxib into polylactide-co-glycolide (PLGA) nanoparticles and to evaluate their antitumor activity in glioma cells. Biodegradable nanoparticles have been extensively investigated in drug delivery systems. ${ }^{16-18}$ Due to their small particle size, they are attracted to their target tissues and have the advantages of targeted drug delivery to the desired site of action, prolonged blood circulation of the encapsulated drug, and reduced drug side effects. ${ }^{19-21} \mathrm{We}$ also investigated the physicochemical properties of PLGA nanoparticles incorporating celecoxib, and their antitumor activity was studied using glioma cell lines. The potential effects of this selective COX-2 inhibitor on glioma cell proliferation, migration, and inhibition of COX-2 expression were also examined in vitro. We expected that selective COX-2 inhibitor and its nanoparticles would be able to inhibit migration and proliferation in glioma cell lines.

\section{Materials and methods \\ Materials}

PLGA was purchased from Boehringher Ingelheim (Ingelheim, Germany). A dialysis membrane with a molecular weight cutoff of 12,000 g/mol was purchased from Spectra/Por (Rancho Dominguez, CA). Dimethylformamide, dimethylacetamide, tetrahydrofuran, dimethylsulfoxide, 1,4-dioxane, and acetone of high-pressure liquid chromatography grade were purchased from Sigma-Aldrich Chemical Company Ltd (St Louis, MO). All other chemicals and reagents were used as extra reagent grade in all experiments.

\section{Preparation of PLGA nanoparticles incorporating celecoxib}

PLGA nanoparticles incorporating celecoxib were prepared as described in a previous report ${ }^{22}$ with brief modification (Figure 1C). When acetone and tetrahydrofuran were used as the preparation solvents, $40 \mathrm{mg}$ of PLGA was dissolved in $7 \mathrm{~mL}$ of solvent, and $5 \mathrm{mg}$ of celecoxib was then added to this solution, which was poured into $10 \mathrm{~mL}$ of deionized water to form nanoparticles and stirred for 15 minutes. The solvent was evaporated using a rotary evaporator (Rotary Vacuum Evaporator, Type N-N, Eyela, Rikakikai Company Ltd, Tokyo, Japan) under reduced pressure for 30 minutes.

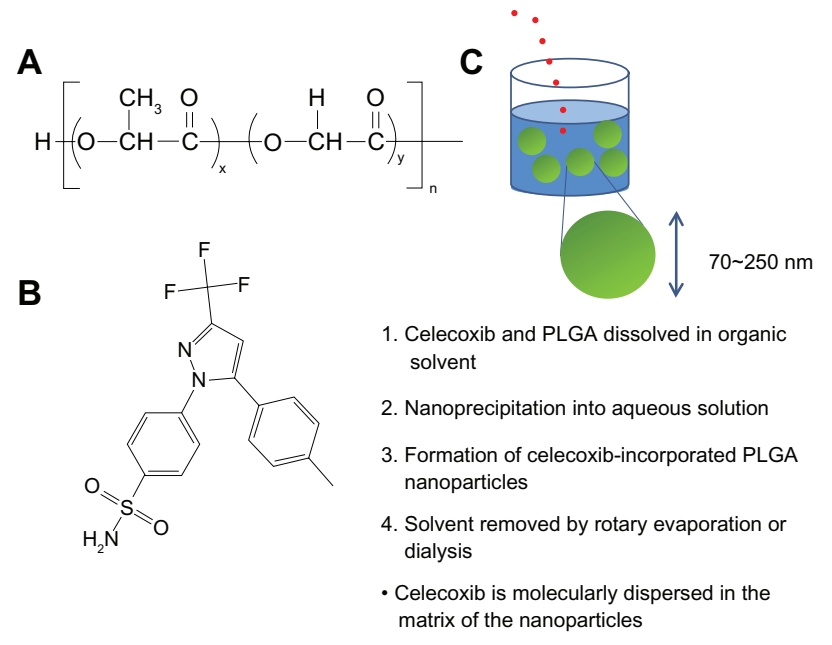

Figure I (A) Chemical structure of PLGA and (B) celecoxib. Schematic illustrations of formation of PLGA nanoparticles incorporating celecoxib by nanoprecipitation. Celecoxib is molecularly dispersed in the matrix of the nanoparticles.

The residual solvent was then removed by a dialysis method (dialysis tube molecular weight cutoff $12,000 \mathrm{~g} / \mathrm{mol}$ ) for 9 hours. The dialyzed solution was harvested, and the volume of nanoparticle solution was adjusted to $40 \mathrm{~mL}$, ie, $1 \mathrm{mg}$ polymer/mL water. This solution was lyophilized and used for analysis.

Using dimethylsulfoxide, dimethylformamide, dimethylacetamide, and 1,4-dioxane as solvents, $40 \mathrm{mg}$ of PLGA and $5 \mathrm{mg}$ of celecoxib were dissolved in $7 \mathrm{~mL}$ of solvent, and were then poured into $10 \mathrm{~mL}$ of deionized water following stirring for 10 minutes. The organic solvent was removed using dialysis tubing for 24 hours. During the dialysis procedure, the deionized water was exchanged every 2 hours. The dialyzed solution was then harvested and the volume of the nanoparticle solution was adjusted to $40 \mathrm{~mL}$. This solution was lyophilized and used for analysis.

Empty PLGA nanoparticles were prepared without addition of celecoxib using dimethylformamide, and the same procedure was then used to make the nanoparticles.

Drug concentration, drug content, and drug loading efficiency was determined by ultraviolet spectrophotometry. ${ }^{23}$ The volume of the dialyzed nanoparticle solution was adjusted to $40 \mathrm{~mL}$ with deionized water (ie, $40 \mathrm{mg}$ of PLGA/40 mL of water), and $100 \mu \mathrm{L}$ of adjusted solution was diluted with dimethylsulfoxide. The celecoxib concentration was measured at $254 \mathrm{~nm}$ using an ultraviolet spectrophotometer (UV1200, Shimadzu, Tokyo, Japan). For the blank test, an empty PLGA nanoparticle solution was adjusted to $40 \mathrm{~mL}$ (ie, $40 \mathrm{mg}$ of PLGA/40 mL of water), and $0.1 \mathrm{~mL}$ of this solution was diluted with dimethylsulfoxide. All experiments 
were performed in triplicate. The equations for drug content and drug loading efficiency are as follows:

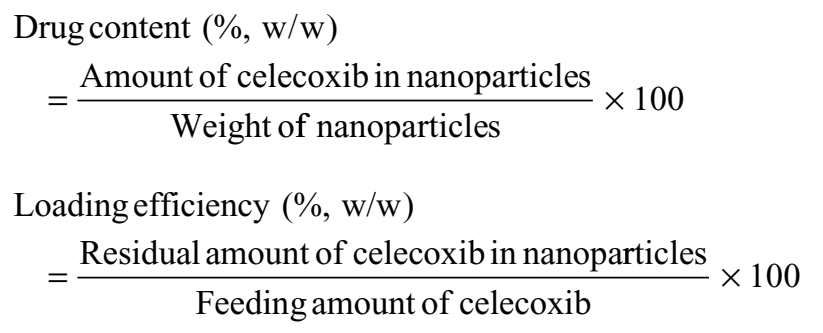

The drug release test was performed as follows: the volume of dialyzed solution was adjusted to $40 \mathrm{~mL}$, and $5 \mathrm{~mL}$ of the adjusted solution was introduced into a dialysis tube. After that, the dialysis tube was put into a bottle with $95 \mathrm{~mL}$ of phosphate-buffered solution. The release test was performed at $37^{\circ} \mathrm{C}$ at a stirring rate of $50 \mathrm{rpm}$. Whole media were discarded at specific time intervals and replaced with fresh phosphate-buffered solution to prevent drug saturation. The amount of celecoxib released was evaluated at $254 \mathrm{~nm}$ by ultraviolet-visible spectrophotometry.

\section{Transmission electron microscopy observations}

For transmission electron microscopy, a drop of nanoparticle suspension containing $0.05 \%(\mathrm{w} / \mathrm{v})$ of phosphotungstic acid was placed on a transmission electron microscopy copper grid coated with carbon film and dried at room temperature. Observation was performed at $80 \mathrm{kV}$ using a JEM-2000 FX II (JEOL, Peabody, MA).

\section{Measurement of particle size}

Measurement of nanoparticle size (concentration $0.1 \mathrm{wt} \%$ ) was performed using photon correlation spectroscopy (Zetasizer 3000; Malvern Instruments, Worcestershire, UK) with a He-Ne laser beam with a wavelength of $633 \mathrm{~nm}$ at $25^{\circ} \mathrm{C}$ (scattering angle of $90^{\circ}$ ).

\section{X-ray powder diffraction}

Crystallinity of the drug and the nanoparticles was determined using X-ray powder diffraction (Rigaku D/Max-1200; Rigaku Americas Corporation, The Woodlands, TX) with Ni-filtered $\mathrm{CuK}$ radiation $(40 \mathrm{kV}, 20 \mathrm{~mA})$. The conditions used for X-ray powder diffraction measurement were as follows: data type, binary; goniometer, 1; attachment, 1; scan mode, continuous; mode $2(\mathrm{R} / \mathrm{T})$, reflection; scan axis, 2-theta/theta; start angle, 10.000; stop angle, 80.000; scan speed, 5.000; sampling interval, 0.050; theta angle, 5.000; 2 theta angle, 10.000; fixed time, 0.01; full scale, 1000; counting unit, CPS; target, $\mathrm{Cu}$; wavelength Ka1 1.540510; wavelength Ka 1.544330; wavelength Ka 1.541780; wavelength Kb 1.39217040 .0 kV and $20.0 \mathrm{~mA}$. Celecoxib, empty PLGA nanoparticles, and PLGA nanoparticles incorporating celecoxib were investigated by X-ray powder diffraction. A $90 \mathrm{mg}$ sample of empty nanoparticles was mixed with $10 \mathrm{mg}$ of celecoxib as a physical mixture.

\section{Cells}

Brain tumor cell lines, including U87MG and C6 rat glioma cells, were obtained from the American Type Culture Collection (Rockville, MD). The cells were maintained in minimum essential medium containing $10 \%$ fetal bovine serum in a $\mathrm{CO}_{2}$ incubator $\left(5 \% \mathrm{CO}_{2}\right.$ at $\left.37^{\circ} \mathrm{C}\right)$.

\section{Western blot assay}

COX-2 expression was checked using C6 rat glioma cells in vitro. ${ }^{9,11,24}$ The cells were grown in $6 \mathrm{~cm}$ culture dishes and treated or not treated with the COX-2 inhibitor for 1 day. The cells were then washed with cold phosphate-buffered solution and homogenized in Tris- $\mathrm{HCl}$ buffer $(\mathrm{pH}$ 7.4) containing $0.5 \%$ NP-40 and protease inhibitors (Boehringer Mannheim, Indianapolis, IN). Samples containing $50 \mu \mathrm{g}$ of protein and sodium dodecyl sulfate polyacrylamide gel electrophoresis loading buffer with $5 \% \alpha$-mercaptoethanol were heated for 5 minutes at $100^{\circ} \mathrm{C}$, and loaded on $8 \%$ polyacrylamide gel (Bio-Rad, Hercules, CA). Electrophoretic transfer to membranes (Millipore, Bedford, MA) was followed by immunoblotting with an antimouse COX-2 antibody (BD Transduction Laboratories, Lexington, KY). This was followed by hybridization using a secondary antibody conjugated with peroxidase (Amersham Pharmacia Biotech, Piscataway, NJ). The signal was detected by chemiluminiscence using the ECL-Plus detection system (Amersham Pharmacia Biotech).

\section{Cell proliferation assay}

The effect of celecoxib and PLGA nanoparticles incorporating celecoxib on cell growth was determined using a MTT cell proliferation assay. ${ }^{25}$ Celecoxib was dissolved in 100\% dimethylsulfoxide as a stock solution $(40 \mathrm{mg} / \mathrm{mL})$ and then diluted 200 times using minimum essential medium. The final dimethylsulfoxide concentration was maintained at less than $0.5 \%(\mathrm{v} / \mathrm{v})$. Dimethylsulfoxide $0.5 \%(\mathrm{v} / \mathrm{v})$ in minimum essential medium was used as a control. PLGA nanoparticles 
incorporating celecoxib were distributed and diluted with minimum essential medium. Glioma cell lines are seeded at a density of $5 \times 10^{3}$ per well in 96-well plates with minimum essential medium containing 10\% fetal bovine serum and incubated overnight in a $\mathrm{CO}_{2}$ incubator $\left(5 \% \mathrm{CO}_{2}\right.$ at $\left.37^{\circ} \mathrm{C}\right)$. After that, fresh medium containing drug or nanoparticles are added. After incubation for the desired period, a MTT Cell Titer 96 cell proliferation assay was performed. Absorbance was measured at $560 \mathrm{~nm}$ using a microtiter plate reader (Thermomax; Molecular Devices, San Jose, CA).

\section{Migration assay}

A migration assay using the U87MG cell line was performed using a simple scratch technique. ${ }^{25}$ Briefly, the cell culture media was replaced with medium containing $5 \mathrm{mM}$ hydroxyurea to eliminate any confounding effects of the experimental agents on cell proliferation. Twenty-four hours of treatment with hydroxyurea $5 \mathrm{mM}$ resulted in complete inhibition of cell proliferation.

After 24 hours of hydroxyurea treatment, the cultures are scraped with a single-edged razor blade. The cells were washed twice with phosphate-buffered solution and placed in medium containing hydroxyurea and celecoxib of various concentrations. After 48 hours of incubation, the cells were washed twice with phosphate-buffered solution, fixed in absolute alcohol, and stained with $0.1 \%$ toluidine blue.

Three microscopic fields were evaluated for each wound injury. The number of cells migrating across the wound edge and the maximum distance migrated (wound edge to nucleus of most distant cell) were determined in each field and averaged for each injury. These experiments were repeated three times.

\section{Results and discussion Characterization of PLGA nanoparticles incorporating celecoxib}

PLGA nanoparticles incorporating celecoxib were prepared by nanoprecipitation and a dialysis method using various solvents, whereby celecoxib and the polymer dissolved in a solvent were precipitated into water and the organic solvent was removed by evaporation or a dialysis procedure (Figure 1C). Nanoprecipitation is a widely used method for nanoparticle preparation. ${ }^{16,26}$ Various factors, such as drug and polymer solubility in an organic solvent, particle size, particle morphology, and aqueous solubility of the organic solvent, can affect drug-loading efficiency. ${ }^{20-24}$ Various solvents were used for preparation of the PLGA nanoparticles incorporating celecoxib to identify the best solvent.
Volatile solvents, such as acetone and tetrahydrofuran, are easily removed by evaporation. However, other solvents, such as dimethylformamide, dimethylsulfoxide, dimethylacetamide, and 1,4-dioxane, are difficult to remove by evaporation, so were removed using a dialysis method. Figure 2A shows the size distribution of the PLGA nanoparticles incorporating celecoxib. As shown in Figure 1, PLGA nanoparticles incorporating celecoxib showed a narrow size distribution of 50-200 $\mathrm{nm}$ and the average particle size was about $92 \mathrm{~nm}$. The morphology of these nanoparticles was observed by transmission electron microscopy, as shown
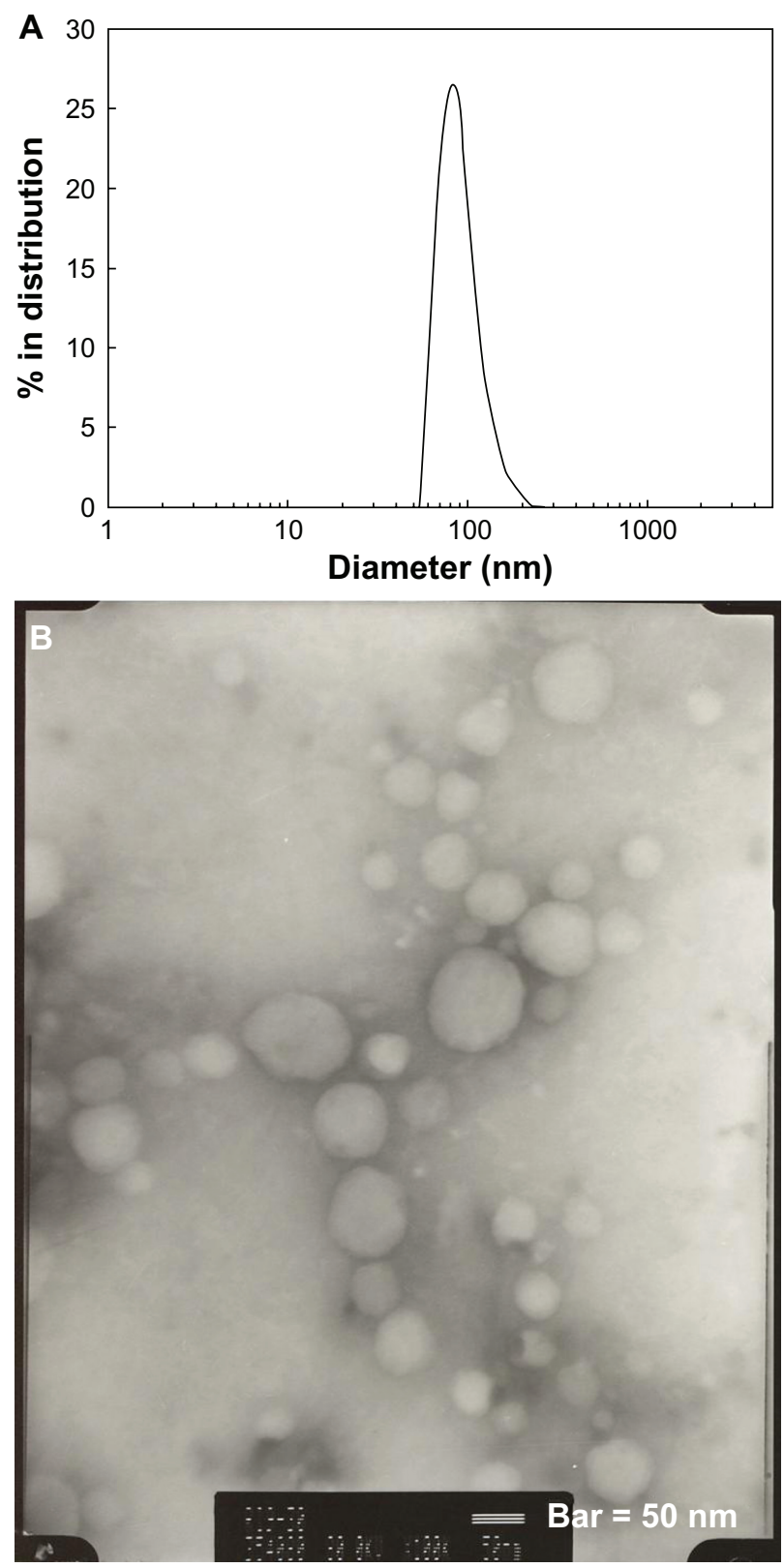

Figure 2 (A) Typical particle size measured by dynamic light scattering and (B) morphology of PLGA nanoparticles incorporating celecoxib (acetone, 40/5 in Table I) observed by transmission electron microscopy. 
Table I Characterization of PLGA nanoparticles incorporating celecoxib according to organic solvent used

\begin{tabular}{|c|c|c|c|c|c|c|c|}
\hline \multirow{2}{*}{$\begin{array}{l}\text { Solvent } \\
\text { used }\end{array}$} & \multirow{2}{*}{$\begin{array}{l}\text { Weight ratio of } \\
\text { PLGA/CEL (mg/mg) }\end{array}$} & \multicolumn{2}{|c|}{ Drug contents $(\%, w / w)$} & \multirow{2}{*}{$\begin{array}{l}\text { Loading } \\
\text { efficiency } \\
(\%, w / w)\end{array}$} & \multicolumn{3}{|l|}{ Particle size (nm) } \\
\hline & & Theoretical & Experimental & & Intensity, mean & Weight, mean & Number, mean \\
\hline \multirow[t]{2}{*}{ Acetone } & $40 / 5$ & II.II & 7.8 & 67.6 & $117.4 \pm 36.1$ & $91.9 \pm 25.2$ & $78.7 \pm 16.4$ \\
\hline & $40 / 10$ & 20.0 & 10.5 & 47.0 & $172.5 \pm 59.1$ & $132.7 \pm 46.3$ & $110.7 \pm 30.1$ \\
\hline THF & $40 / 5$ & II.II & 6.3 & 54.0 & $255.5 \pm 63.5$ & $213.6 \pm 51.7$ & $186.5 \pm 37.2$ \\
\hline DMSO & $40 / 5$ & 11.11 & 3.8 & 31.6 & $105.0 \pm 64.0$ & $80.5 \pm 20.5$ & $70.3 \pm 7.6$ \\
\hline DMAc & $40 / 5$ & II.II & 4.2 & 35.1 & $150.7 \pm 1 \mid 3.3$ & $100.7 \pm 70.6$ & $87.4 \pm 29.2$ \\
\hline I,4-dioxane & $40 / 5$ & 11.11 & 4.4 & 36.8 & $164.5 \pm 58.5$ & $125.4 \pm 39.3$ & $102.3 \pm 24.3$ \\
\hline DMF & $40 / 5$ & II.II & 4.2 & 35.1 & $\mid 15.1 \pm 32.6$ & $96.8 \pm 29.3$ & $80.7 \pm 21.4$ \\
\hline
\end{tabular}

Abbreviations: CEL, celecoxib; DMSO, dimethylsulfoxide; DMF, dimethylformamide; DMAc, dimethylacetamide; THF, tetrahydrofuran.

in Figure 2B, and they were found to have spherical shapes and particle sizes around $100 \mathrm{~nm}$. This result is similar to the particle size data shown in Figure 2A.

The effects of various solvents on nanoparticle formation are summarized in Table 1. Changes in the solvent used had little effect on the physicochemical properties of the nanoparticles, such as particle size and drug loading efficiency. The size of the PLGA nanoparticles incorporating celecoxib was about $100 \mathrm{~nm}$ for almost all of the samples. However, the size of the nanoparticles increased when tetrahydrofuran was used as the solvent. When the feeding amount of drug was increased, the particle size also increased slightly. Because the distribution of nanoparticles in the body is significantly influenced by particle size, ${ }^{17,27}$ small-sized nanoparticles are preferred for intravenous administration. In particular, particles smaller than $200 \mathrm{~nm}$ are considered acceptable for passive drug targeting. ${ }^{27,28}$

When acetone and tetrahydrofuran was used as the solvents, drug content and loading efficiency were relatively higher than for the other solvents, possibly because the drug is easily liberated from dialysis tubing during the dialysis procedure, whereas nonvolatile solvents, such as dimethylformamide, dimethylsulfoxide, dimethylacetamide, and 1,4-dioxane, require a longer period of dialysis to remove the organic solvent. The drawbacks of the dialysis method are the ease of drug release from the dialysis membrane before and after nanoparticle formation. When the drug feeding amount was increased, the drug content also increased, but the drug loading efficiency decreased.

Figure 3 shows the X-ray powder diffraction data for PLGA nanoparticles incorporating celecoxib. Celecoxib showed intrinsic crystalline peaks (Figure 3A), while the empty nanoparticles showed broad peak properties (Figure 3B). PLGA nanoparticles incorporating celecoxib had peak characteristics very similar to those of the empty nanoparticles (Figure 3C), indicating that drug was
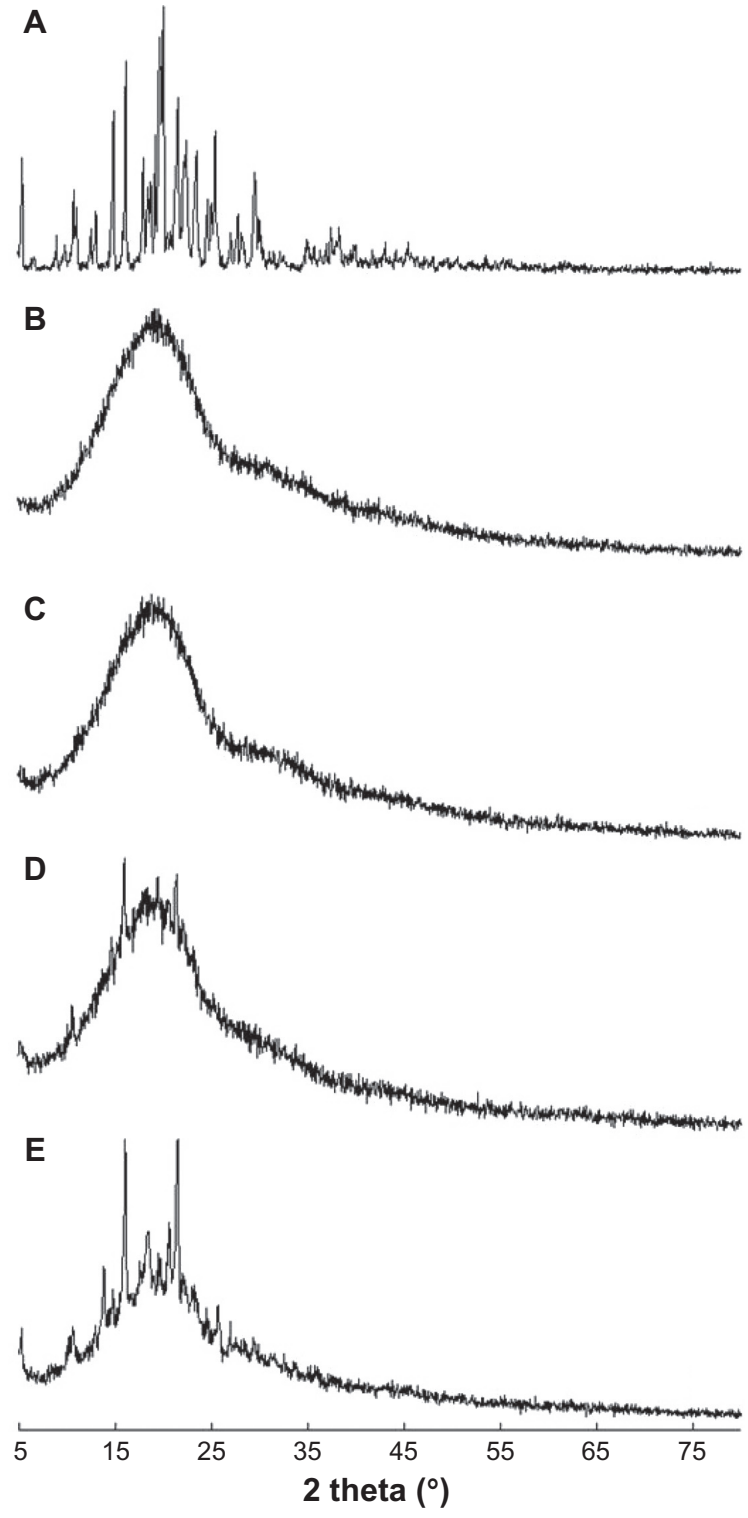

Figure 3 X-ray powder diffraction patterns for PLGA nanoparticles incorporating celecoxib. (A) Celecoxib, (B) empty nanoparticles, (C) PLGA nanoparticles incorporating celecoxib (drug content 7.8\%, w/w), (D) drug content $10.5 \%, \mathrm{w} / \mathrm{w}$, and $(\mathbf{E})$ a physical mixture of celecoxib and empty nanoparticles $(1 / 10, \mathrm{w} / \mathrm{w})$. Note: The data show that celecoxib was molecularly dispersed in the nanoparticle matrix at a lower drug content, and aggregated or crystallized at a higher drug content. 

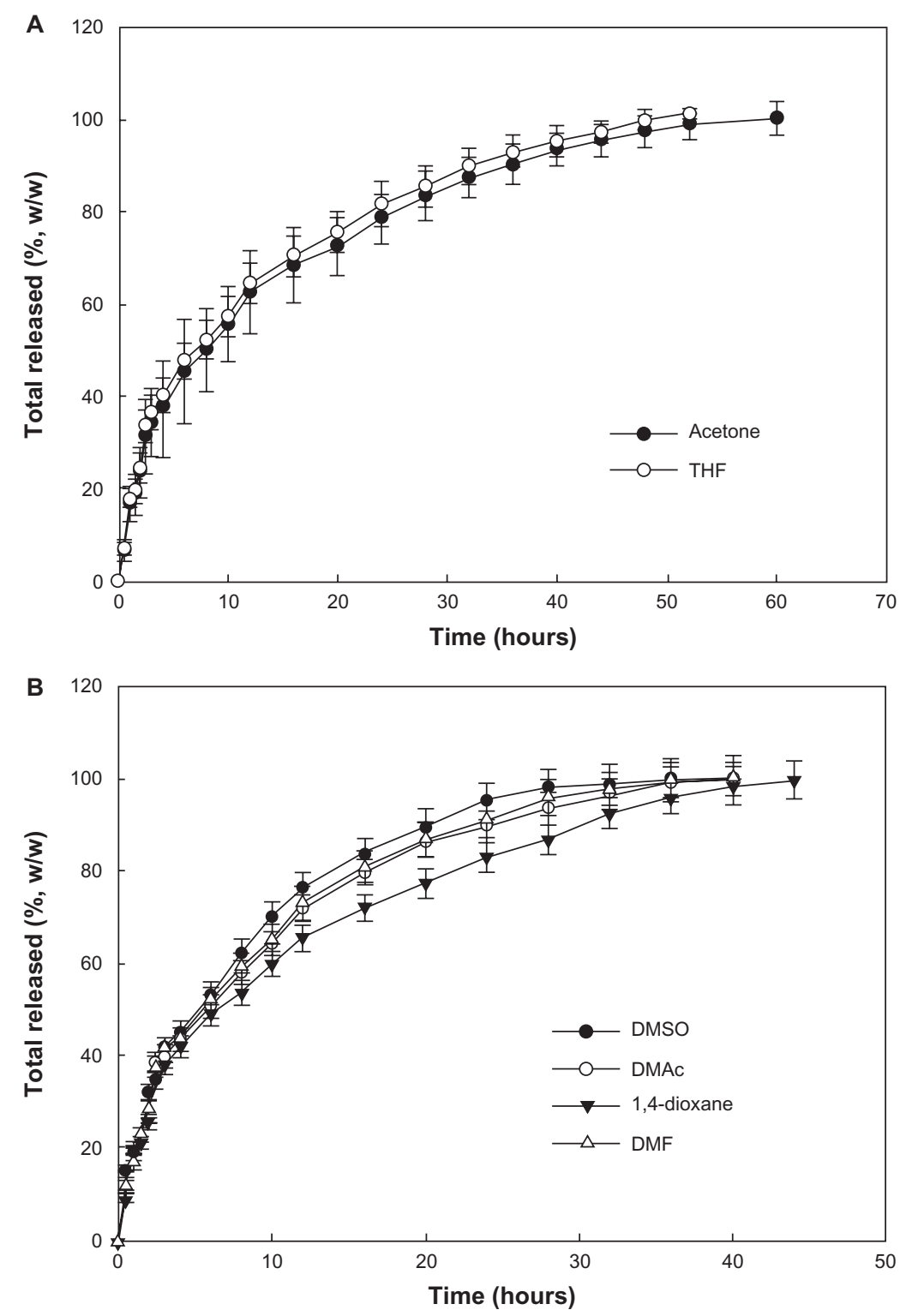

Figure 4 Effect of solvent used on drug release from the PLGA nanoparticles. Solvents used for preparation of the nanoparticles were (A) acetone and tetrahydrofuran and (B) dimethylsulfoxide, dimethylacetamide, I,4-dioxane, and dimethylformamide. The properties of the PLGA nanoparticles are shown in Table I.

Abbreviations: THF, tetrahydrofuran; DMSO, dimethylsulfoxide; DMAc, dimethylacetamide; DMF, dimethylformamide.

incorporated inside the nanoparticles. However, when the feeding amount of drug was increased, drug crystalline peaks were observed (Figure 3D), indicating that some of the free drug might have been crystallized in the matrix of the nanoparticles. As shown in Figure 3E, a physical mixture of empty nanoparticles and drug showed intrinsic peaks for both the empty nanoparticles and the drug. At low content, the drug could exist as a molecular dispersion in the nanoparticle matrix and its intrinsic crystalline peaks would disappear. However, drug can be aggregated at a higher drug loading, and crystalline peaks can be observed. ${ }^{19}$ Tan et al reported on a silica-lipid hybrid incorporating celecoxib microcapsules for oral drug delivery. ${ }^{29}$ They described similar results for $\mathrm{X}$-ray powder diffraction, ie, the microcapsules incorporating celecoxib showed a broad spectrum, while the drug itself showed sharp intrinsic crystalline peaks.

\section{Drug release study}

Figure 4 shows the effect of the solvent used on release of celecoxib from the nanoparticles. An initial burst was observed for about 4 hours, and then the drug was released in a controlled manner over a period of 2 days. When acetone or tetrahydrofuran was used as the preparation solvent, celecoxib was released at a slower rate than with the other solvents. This finding might be due to the fact that the drug content achieved using acetone and tetrahydrofuran was higher than for the other solvents. Generally, a hydrophobic drug can be crystallized into nanoparticles, thereby achieving a higher 
drug content. ${ }^{19}$ The release rate for a crystallized drug in nanoparticles is relatively slow compared with that for a molecular dispersion of drug in nanoparticles. At a lower drug content, the drug can exist as a molecular dispersion, and crystalline peaks for celecoxib are not seen on X-ray powder diffraction measurement. Particle size is another factor affecting drug release kinetics. It has been reported that larger nanoparticles have a slower drug release rate than smaller ones. ${ }^{16,20}$ Figure 5 shows the effect of drug content on its release rate from nanoparticles. The higher the drug content, the slower the release rate of the drug. At higher drug feeding, the drug can be crystallized in the nanoparticles, and this was supported by our X-ray powder diffraction findings.

Tan et al reported that the drug release rate can be changed by concentration of the surfactant, using sodium lauryl sulfate and other formulation materials. They reported that silicalipid hybrid microcapsules were effective for enhancing drug dissolution properties and increasing the drug half-life in plasma. ${ }^{29}$ Thakkar et al reported that celecoxib incorporated into albumin microspheres was released over about 1 week. ${ }^{23}$ Furthermore, they also observed an initial burst effect for 12 hours, ie, more than $50 \%(\mathrm{w} / \mathrm{w})$ of the drug was released in the first 12 hours. Ayalasomayajula and Kompella reported on their construction of PLGA microspheres containing celecoxib using an oil/water emulsion solvent evaporation method. ${ }^{30}$ They reported that celecoxib was released from the microspheres over 49 days, and that less than $40 \%$ of the drug was released during this period. However, a mean nanoparticle diameter smaller $300 \mathrm{~nm}$ may enable faster drug release than from microspheres, ie, more than $80 \%(\mathrm{w} / \mathrm{w})$ of the drug was released from PLGA nanoparticles incorporating celecoxib. ${ }^{31}$ In our experiment, drug release continued over 1 day, suggesting that drug release from nanoparticles can change significantly according to the polymer properties, particle size, and particle morphology. Nanoparticles incorporating celecoxib were successively prepared during this experiment, and we used celecoxib nanoparticles prepared from acetone in a subsequent study (drug content, 10.5\%, w/w).

\section{Antitumor activity of PLGA nanoparticles incorporating celecoxib}

COX-2 expression in the brain is enhanced in the presence of inflammatory disorders, trauma, ischemia, and Alzheimer's disease. ${ }^{32-35}$ It has been reported that COX-2-positive cells accumulate in areas of necrosis. ${ }^{36,37}$ This could represent induction of COX-2 in tumor cells by hypoxia or by factors released at the sites of necrosis. Increased levels of prostaglandins are due to COX-2 overexpression. This has been previously reported in colon and human brain tumor tissues when compared with normal tissue, ${ }^{36-38}$ suggesting that prostaglandins play a role in tumor development. Thus, COX-2 inhibitors may lead to a reduction in prostaglandin levels which, in turn, may inhibit proliferation and migration, and induce apoptosis in glioma cell lines. A similar effect has been reported in colon, esophageal, and pancreatic carcinoma cell lines, where a selective COX-2 inhibitor was shown to inhibit proliferation and to induce apoptosis..$^{11-13,39,40}$ Treatment of brain tumors using celecoxib, a selective COX-2 inhibitor, is expected to improve the treatment of this type of tumor, as well as improve quality of life for patients.

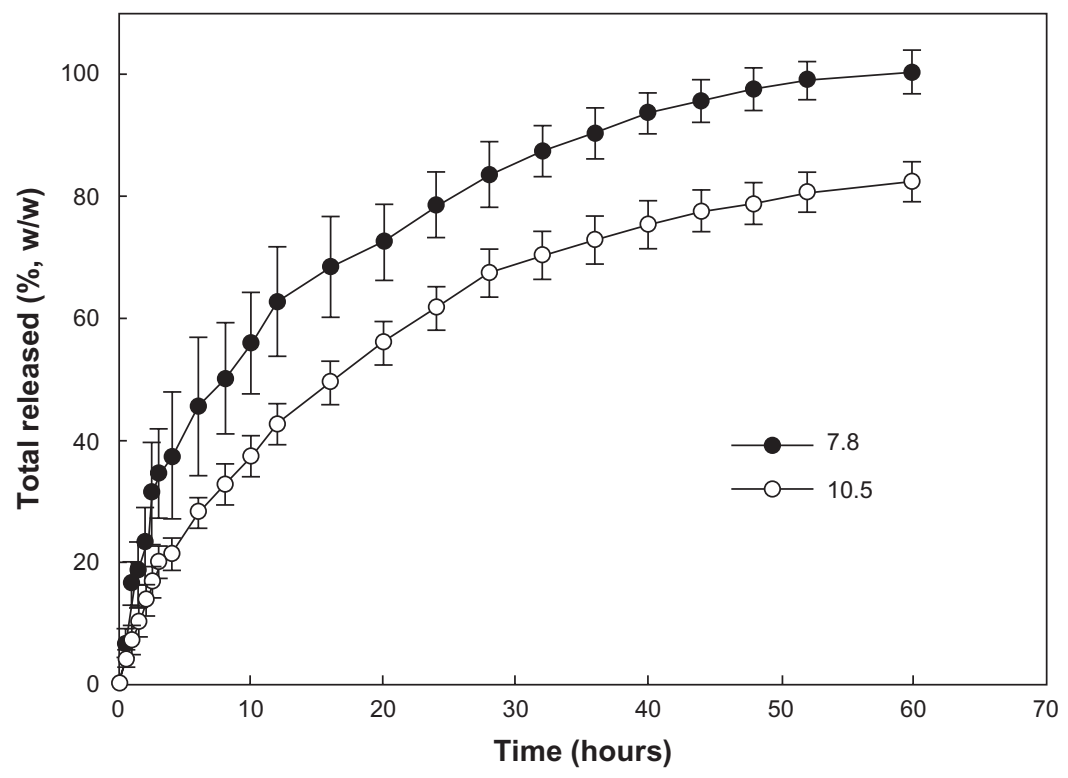

Figure 5 Effect of drug contents on celecoxib release from the PLGA nanoparticles. 
To evaluate the antitumor activity of celecoxib, glioma cells were treated with PLGA nanoparticles incorporating celecoxib or celecoxib alone. As shown in Figure 6, celecoxib and PLGA nanoparticles incorporating celecoxib showed similar cytotoxicity, whereas empty nanoparticles did not affect
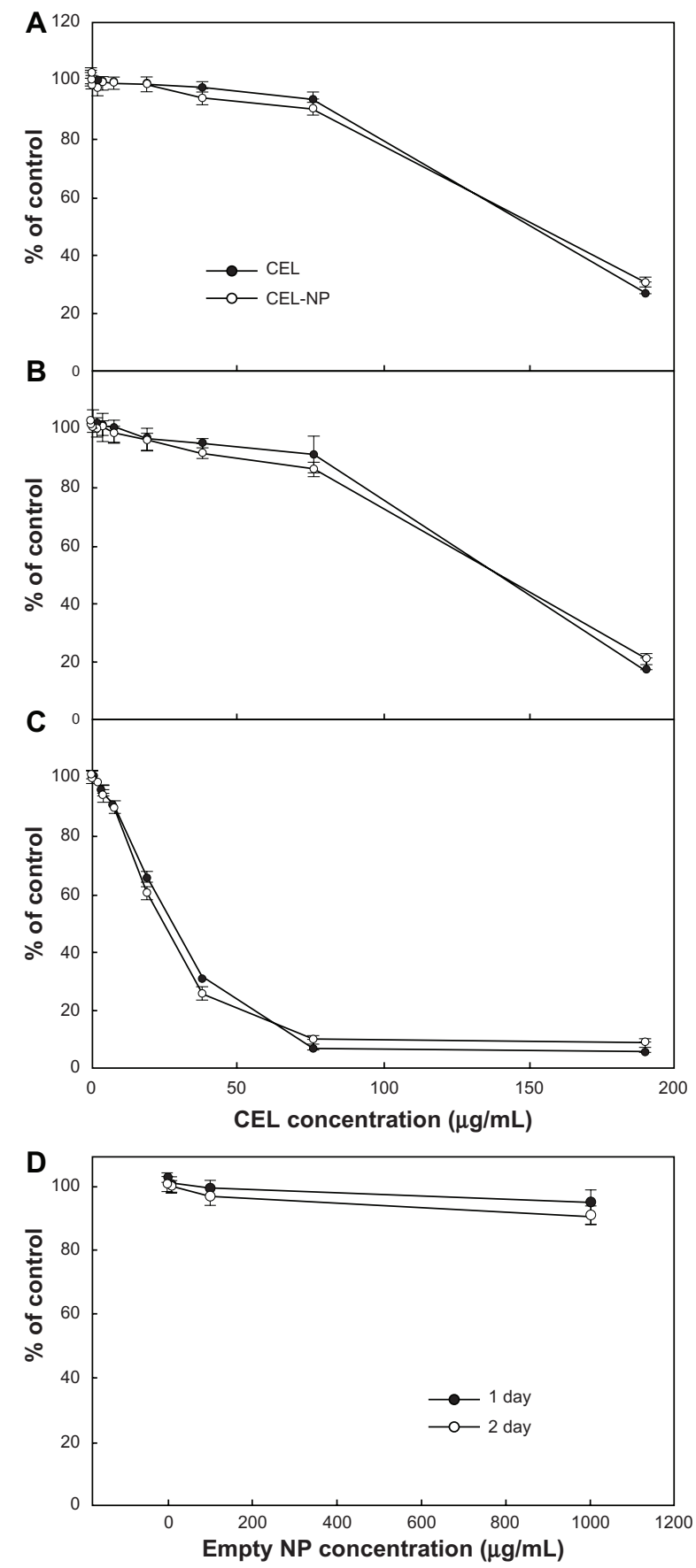

Figure 6 Cytotoxicity of PLGA nanoparticles incorporating celecoxib against brain tumor cells. Different concentrations of celecoxib and PLGA nanoparticles incorporating celecoxib were treated for (A) I day, (B) 2 days, and (C) 4 days against U87 tumor cells.

Notes: Data points represent the mean from eight replicate wells and the bars indicate the standard deviation of the mean. Values were calculated relative to the control ( $0.1 \%$ dimethylsulfoxide). Absorbance of sample/absorbance of control $\times 100$. tumor cell growth. These results indicate that nanoparticles incorporating celecoxib have similar antitumor activity to that of celecoxib alone. The effect of celecoxib on migration was studied in a two-dimensional culture system, as shown in Figure 7. U87MG cells were grown to confluence in a $6 \mathrm{~cm}$ culture dish, and half of the cultured cell area was removed following treatment overnight with hydroxyurea, then exposed to different concentrations of celecoxib. Figure 7A shows images of tumor cell migration and Figure 7B shows the migration distance. Migration of U87MG cells in this two-dimensional system was not significantly changed by any concentration of celecoxib, while cell growth was slightly changed at higher concentrations. These results indicate that migration of U87MG cells is not significantly inhibited by COX-2 inhibition, even though cell proliferation was affected by treatment with celecoxib.

Figure 8 shows the effect of celecoxib and PLGA nanoparticles incorporating celecoxib on the growth of $\mathrm{C} 6$ rat glioma cells. Both celecoxib and PLGA nanoparticles incorporating celecoxib showed similar cytotoxicity against C6 cells in a dose-dependent manner. Empty PLGA nanoparticles did not affect tumor cell growth. A Western blot assay was used to investigate whether or not celecoxib inhibited COX-2 expression in C6 cells. As shown in Figure 9, COX-2 expression in $\mathrm{C} 6$ cells was not significantly suppressed by treatment with celecoxib or the nanoparticles. In other reports, expression of COX-2 protein was also not specifically inhibited by celecoxib. ${ }^{41,42}$ Barnes et al ${ }^{41}$ reported that COX-2 protein expression was decreased by treatment with celecoxib. However, it seems that the decreased COX-2 expression and antiproliferative effect of celecoxib treatment is more related to apoptosis of tumor cells rather than specific inhibition of COX-2 expression by celecoxib. ${ }^{41,42}$ Celecoxib is known to inhibit expression of non-COX-2 targets, such as carbonic anhydrases, 3-phosphoinositide-dependent protein kinase-1, sarcoplasmic/endoplasmic reticulum, and calcium ATPase in tumor cells. Furthermore, celecoxib is known to increase apoptosis of tumor cells via inactivation of Akt, which is a member of the serine/threonine kinase family. Our results indicate that inhibition of proliferation of tumor cells by celecoxib is not closely related to COX-2 expression. Even if celecoxib or PLGA nanoparticles incorporating celecoxib did not significantly affect COX-2 expression and tumor cell mobility, nanoparticles had the same antiproliferative effect on brain tumor cells.

To test the stability of celecoxib in the nanoparticle matrix, PLGA nanoparticles incorporating celecoxib was recovered at 24 hours in the drug release experiment and used 

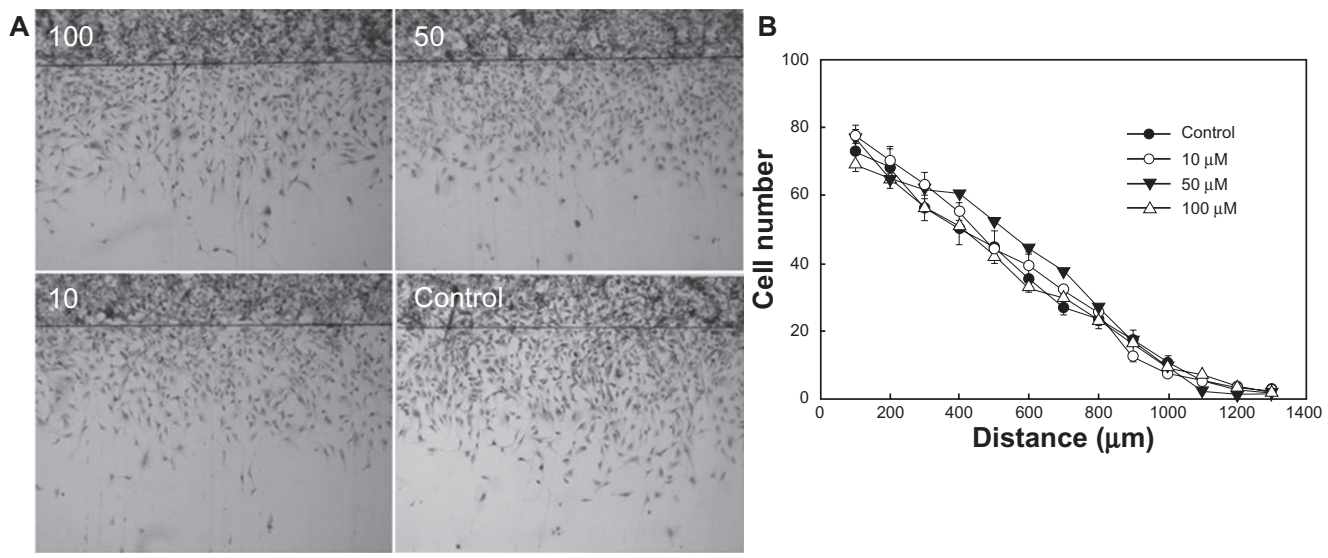

Figure 7 (A) Effect of various concentrations of celecoxib on migration of U87MG cells (in $\mu$ M). U87MG cells confluently filled a $6 \mathrm{~cm}$ culture dish, and half of the area of the cultured cells was removed by a knife following overnight treatment with hydroxyurea and exposed to different concentrations of celecoxib. (B) Comparison of migration ability in an in vitro assay after treatment with a range of celecoxib concentrations.
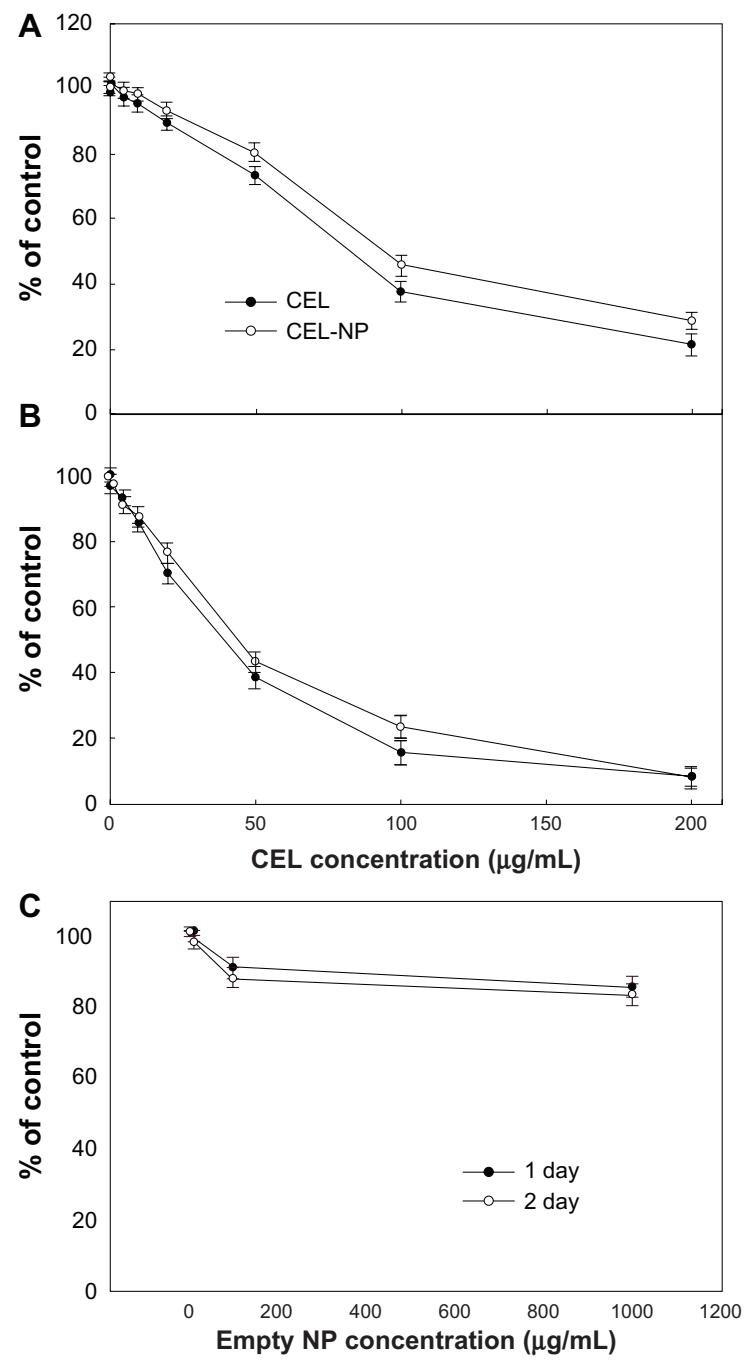

Figure 8 Cytotoxicity of PLGA nanoparticles incorporating celecoxib against C6 rat glioma cells. Different concentrations of celecoxib and PLGA nanoparticles incorporating celecoxib were used for (A) I day and (B) 2 days to treat $\mathrm{C} 6$ tumor cells.

Notes: Data points represent the mean for eight replicate wells and bars indicate the standard deviation of the mean. The value was calculated relative to control ( $0.1 \%$ dimethylsulfoxide). Absorbance of sample/absorbance of control $\times 100$.

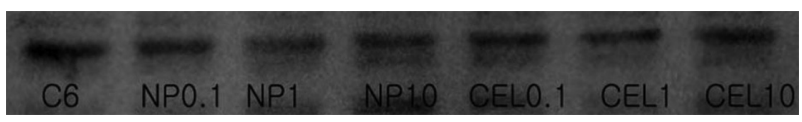

Figure 9 Western blot analysis for COX-2 protein in C6 rat glioma cells. Each lane is as follows: C6 cells treated with celecoxib or PLGA nanoparticles incorporating celecoxib.
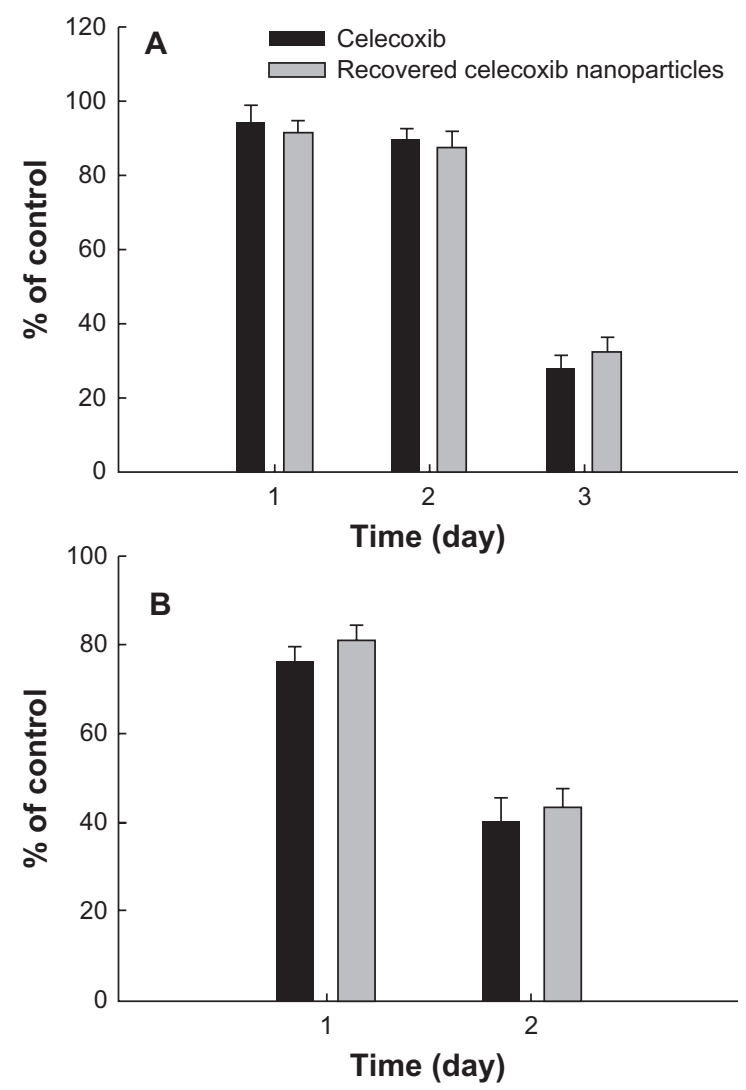

Figure 10 Cytotoxicity of PLGA nanoparticles incorporating celecoxib recovered from drug release experiment against U87MG cells (A) and C6 rat glioma cells (B). During the drug release experiment, PLGA nanoparticles incorporating celecoxib were harvested at 24 hours and dissolved in dimethylsulfoxide, and the harvested celecoxib solution was diluted with cell culture medium. A calculated amount $(50 \mu \mathrm{g} / \mathrm{mL})$ of celecoxib was used to treat the tumor cells. 
to treat the tumor cells, as shown in Figure 10. The celecoxib recovered in the nanoparticles showed similar antitumor activity against U87MG cells (Figure 10A) and C6 cells (Figure 10B), indicating that the intrinsic stability and antitumor activity of celecoxib was not significantly changed during the drug release experiment. The results of our study show that PLGA nanoparticles incorporating celecoxib are promising candidates for antitumor drug delivery.

\section{Conclusion}

PLGA nanoparticles incorporating celecoxib were prepared for antitumor drug delivery. PLGA nanoparticles incorporating celecoxib had spherical shapes and their particle sizes were in the range of 50-200 $\mathrm{nm}$. Drug-loading efficiency was not significantly altered according to the solvent used, except for acetone. The drug was released for longer than 2 days from PLGA nanoparticles. The higher the drug content, the longer the duration of drug release. PLGA nanoparticles incorporating celecoxib showed almost the same cytotoxicity against U87MG tumor cells as celecoxib itself. Furthermore, celecoxib did not affect the degree of migration of U87MG cells. When C6 rat glioma cells were used, PLGA nanoparticles incorporating celecoxib showed dose-dependent cytotoxicity similar to that of celecoxib itself. Neither celecoxib nor PLGA nanoparticles incorporating celecoxib affected COX-2 expression in C6 cells on Western blot assay. In conclusion, PLGA nanoparticles incorporating celecoxib have similar antitumor activity to celecoxib itself, even if they did not affect the degree of migration or COX-2 expression in tumor cells.

\section{Acknowledgment}

This research was supported by Leading Foreign Research Institute Recruitment Program through the National Research Foundation of Korea (NRF) funded by the Ministry of Education, Science and Technology (MEST) (2011-0030034).

\section{Disclosure}

The authors report no conflicts of interest in this work.

\section{References}

1. Smith WL, Garavito M, DeWitt DL. Prostaglandin endoperoxide H synthase (cyclooxygenase)-1 and 2. J Biol Chem. 1996;271(52): 33157-33160.

2. Kargman SL, O'Neill GP, Vickers PJ, Evans JF, Mancini JA, Jothy S. Expression of prostaglandin $\mathrm{G} / \mathrm{H}$ synthase-1 and -2 protein in human colon cancer. Cancer Res. 1995;55(12):2556-2559.

3. Liu XH, Rose DP. Differential expression and regulation of cyclooxygenase- 1 and -2 in two human breast cancer cell lines. Cancer Res. 1996; 56(22):5125-5127.
4. Muller-Decker K, Furstenberger G. The cyclooxygenase-2-mediated prostaglandin signaling is causally related to epithelial carcinogenesis. Mol Carcinog. 2007;46(8):705-710.

5. Restimaki A, Honkanen N, Jankala H, Sipponen P, Harkonen M. Expression of cyclooxygenase-2 in human gastric carcinoma. Cancer Res. 1997;57(7):1276-1280.

6. Sano H, Kawahito Y, Wilder RL, et al. Expression of cyclooxygenase-1 and -2 in human colorectal cancer. Cancer Res. 1995;55(17): 3785-3789.

7. Wilson KT, Fu S, Ramanujam KS, Meltzer SJ. Increased expression of inducible nitric oxide synthase and cyclooxygenase-2 in Barrett's esophagus and associated adenocarcinomas. Cancer Res. 1998;58(14): 2929-2934.

8. Wolff H, Saukkonen K, Anttila S, Karjalainen A, Vainio H, Ristimaki A. Expression of cyclooxygenase-2 in human lung carcinoma. Cancer Res. 1998;58(22):4997-5001.

9. Deininger MH, Weller M, Streffer J, Mittelbronn M, Meyermann R. Patterns of cyclooxygenase- 1 and 2 expression in human gliomas in vivo. Acta Neuropathol. 1999;98(3):240-244.

10. Tsujii M, Kawano S, Tsuji S, Sawaoka H, Hori M, DuBois RN. Cyclooxygenase regulates angiogenesis induced by colon cancer cells. Cell. 1998;93(5):705-716.

11. Molina MA, Sitja-Arnau M, Lemoine MG, Frazier ML, Sinicrope FA. Increased cyclooxygenase-2 expression in human pancreatic carcinomas and cell lines: growth inhibition by nonsteroidal anti-inflammatory drugs. Cancer Res. 1999;59(17):4356-4362.

12. Elder DJ, Halton DE, Hague A, Paraskeva C. Induction of apoptotic cell death in human colorectal carcinoma cell lines by a cyclooxygenase- 2 (COX-2) selective nonsteroidal anti-inflammatory drug: independence from COX-2 protein expression. Clin Cancer Res. 1997;3(10): 1679-1683.

13. Liu XH, Yao S, Kirschenbaum A, Levine AC. NS-398, a selective cyclooxygenase-2 inhibitor, induces apoptosis and down-regulates bcl-2 expression in LNCaP cells. Cancer Res. 1998;58(19):4245-4249.

14. Castelli MG, Chiabrando C, Fanelli R, et al. Prostaglandin and thromboxane synthesis by human intracranial tumors. Cancer Res. 1989;49(6):1505-1508.

15. Paoletti P, Chiabrando C, Gaetani P, Butti MG, Martelli L, Rolli M. Prostaglandins in human brain tumors. J Neurosurg Sci. 1989; 33(1):65-69.

16. Allemann E, Gurny R, Doelker E. Drug-loaded nanoparticles preparation methods and drug targeting issues. Eur J Pharm Biopharm. 1993;39:173-191.

17. Redhead HM, Davis SS, Illum L. Drug delivery in poly(lactide-coglycolide) nanoparticles surface modified with poloxamer 407 and poloxamine 908: in vitro characterisation and in vivo evaluation. J Control Release. 2001;70(3):353-363.

18. Davis SS, Illum L, Moghimi SM, et al. Microspheres for targeting drugs to specific body sites. J Control Release. 1993;24(1-3): 157-163.

19. Gref R, Minamitake Y, Peracchia MT, Trubetskoy V, Torchilin V, Langer R. Biodegradable long-circulating polymeric nanospheres. Science. 1994;263(5153):1600-1603.

20. Leroux JC, Allemann E, Jaeghere FD, Doelker E, Gurny R. Biodegradable nanoparticles - from sustained release formulations to improved site specific drug delivery. J Control Release. 1996;39(2-3): 339-350.

21. McCarron PA, Marouf WM, Donnelly RF, Scott C. Enhanced surface attachment of protein-type targeting ligands to poly(lactide-coglycolide) nanoparticles using variable expression of polymeric acid functionality. J Biomed Mat Res A. 2008;87(4):873-884.

22. Jeong YI, Kang MK, Sun HS, et al. All-trans retinoic acid release from core-shell type nanoparticles of poly(epsilon-caprolactone)/ poly(ethylene glycol) diblock copolymer. Int J Pharm. 2004;273(1-2): 95-107.

23. Thakkar H, Sharma RK, Mishra AK, Chuttani K, Murthy RR. Albumin microspheres as carriers for the antiarthritic drug celecoxib. AAPS Pharm Sci Tech. 2005;6(1):E65-E73. 
24. Nakatani K, Nakahata N, Arakawa T, Yasuda H, Ohizumi Y. Inhibition of cyclooxygenase and prostaglandin E2 synthesis by gamma-mangostin, a xanthone derivative in mangosteen, in C6 rat glioma cells. Biochem Pharm. 2002;63(1):73-79.

25. Jeong YI, Kim SH, Jung TY, et al. Polyion complex micelles composed of all-trans retinoic acid and poly (ethylene glycol)-grafted-chitosan. J Pharm Sci. 2006;95(11):2348-2360.

26. Govender T, Stolnik S, Garnett MC, Illum L, Davis SS. PLGA nanoparticles prepared by nanoprecipitation: drug loading and release studies of a water soluble drug. J Control Release. 1999;57(2): 171-185.

27. Seijo B, Fattal E, Roblot-Treupel L, Couvreur P. Design of nanoparticles of less than $50 \mathrm{~nm}$ diameter: preparation, characterization and drug loading. Int J Pharm. 1990;62(1):1-7.

28. Scholes PD, Coombes AGA, Illum L, Davis SS, Vert M, Davies MC. The preparation of sub-200 nm poly(lactide-co-glycolide) microspheres for site-specific drug delivery. J Control Release. 1993;25(1-2): $145-153$.

29. Tan A, Simovic S, Davey AK, Rades T, Prestidge CA. Silica-lipid hybrid (SLH) microcapsules: a novel oral delivery system for poorly soluble drugs. J Control Release. 2009;134(1):62-70.

30. Ayalasomayajula SP, Kompella UB. Subconjunctivally administered celecoxib-PLGA microparticles sustain retinal drug levels and alleviate diabetes-induced oxidative stress in a rat model. Eur J Pharm. 2005; 511(2-3):191-198.

31. Mccarron PA, Donnelly RF, Marouf W. Celecoxib-loaded poly(DLlactide-co-glycolide) nanoparticles prepared using a novel and controllable combination of diffusion and emulsification steps as part of the salting-out procedure. J Microencapsul. 2006;23(5):480-498.

32. Lacroix S, Rivest S. Effect of acute systemic inflammatory response and cytokines on the transciption of the genes encoding cyclooxygenase enzymes (COX-1 and COX-2) in the rat brain. J Neurochem. 1998; $70(2): 452-460$.
33. Mittinen S, Fusco FR, Yrjanheikki J, et al. Spreading depression and focal brain ischemia induce cyclooxygenase-2 in cortical neurons through N-methy-D-aspartic acid-receptors and phopholipase A2. Proc Natl Acad Sci U S A. 1997;94(12):6500-6505.

34. Sairanen T, Ristimaki A, Karjalainen-Lindsberg ML, Paetau A, Kaste M, Lindsberg PJ. Cyclooxygenase-2 is induced globally in infarcted human brain. Ann Neurol. 1998;43(6):738-747.

35. Lukiw WJ, Bazan NG. Cyclooxygenase-2 RNA message abundance, stability, and hypervariability in sporadic Alzheimer neocortex. J Neurosci Res. 1997;50(6):937-945.

36. Bennett A, Tacca MD, Stamford IF, Zebro T. Prostaglandins from tumors of human large bowel. Br J Cancer. 1977;35(6):881-884.

37. Maxwell WJ, Kelleher D, Keating JJ, et al. Enhanced secretion of prostaglandin E2 by tissue-fixed macrophages in colonic carcinoma. Digestion. 1990;47(3):160-166.

38. Kokoglu E, TuterY, Sandikci KS, et al. Prostaglandin E2 levels in human brain tumor tissues and arachidonic acid levels in the plasma membrane of human brain tumors. Cancer Lett. 1998;132(1-2):17-21.

39. Williams CS, Luongo C, Radhika A, et al. Elevated cyclooxygenase-2 levels in Min mouse adenomas. Gastroenterology. 1996;111(4) 1134-1140.

40. Zimmerman KC, Sarbia M, Weber AA, Borchard F, Gabbert HE, Schror K. Cyclooxygenase-2 expression in human esophageal carcinoma. Cancer Res. 1999;59(1):198-204.

41. Barnes NL, Warnberg F, Farnie G, et al. Cyclooxygenase-2 inhibition: effects on tumour growth, cell cycling and lymphangiogenesis in a xenograft model of breast cancer. Br J Cancer. 2007;96(4):575-582.

42. Schönthal AH. Direct non-cyclooxygenase-2 targets of celecoxib and their potential relevance for cancer therapy. Br J Cancer. 2007;97(11): 1465-1468.
International Journal of Nanomedicine

\section{Publish your work in this journal}

The International Journal of Nanomedicine is an international, peerreviewed journal focusing on the application of nanotechnology in diagnostics, therapeutics, and drug delivery systems throughou the biomedical field. This journal is indexed on PubMed Central, MedLine, CAS, SciSearch $₫$, Current Contents $₫ /$ Clinical Medicine,

\section{Dovepress}

Journal Citation Reports/Science Edition, EMBase, Scopus and the Elsevier Bibliographic databases. The manuscript management system is completely online and includes a very quick and fair peer-review system, which is all easy to use. Visit http://www.dovepress.com/ testimonials.php to read real quotes from published authors. 